DO-TH 98/04

$\mathrm{DTP} / 98 / 22$

April 1998

\title{
Next-to-Leading Order QCD Corrections to the Polarized Photoproduction of Heavy Flavors
}

\author{
I. Bojak \\ Institut für Physik, Universität Dortmund, D-44221 Dortmund, Germany \\ and \\ M. Stratmann \\ Department of Physics, University of Durham, Durham DH1 3LE, England
}

\begin{abstract}
We present a calculation of the next-to-leading order $\left(\mathcal{O}\left(\alpha_{s}^{2} \alpha\right)\right)$ QCD corrections to heavy flavor photoproduction with longitudinally polarized beams. We apply our results to study the longitudinal spin asymmetry for the total charm quark production cross section which will be utilized by the forthcoming COMPASS experiment at CERN to obtain first direct information on the polarized gluon density $\Delta g$. We also briefly discuss the main theoretical uncertainties inherent in this calculation. In particular we demonstrate that the factorization scale dependence is considerably reduced in next-to-leading order.
\end{abstract}


Despite significant experimental progress in spin-dependent deep inelastic scattering (DIS) [1], the polarized parton densities $\Delta f(f=q, \bar{q}, g)$ still remain considerably less known than their unpolarized counterparts $f$. In particular the polarized gluon density $\Delta g$ is almost completely unconstrained in all recent next-to-leading order (NLO) analyses [2, 3, 4] of presently available DIS data. This is due to the limited kinematical coverage of the data which does not allow for an unambiguous determination of $\Delta g$ from scaling violations. An even more important role plays the lack of a momentum sum rule and the absence of any direct constraints from other processes like jet or direct photon production, both being available and important for the determination of unpolarized parton densities. Therefore a series of 'next generation' spin experiments will put a special emphasis on such exclusive measurements to provide further invaluable information for a more restrictive analysis of polarized parton distributions in the future.

The first direct information on $\Delta g$ is expected to be provided by the COMPASS fixed target experiment at CERN [5] from studies of the longitudinal spin asymmetry of total open charm photoproduction. Heavy quark $(Q=c, b)$ production is generally considered to be one of the best options to pin down $\Delta g$ since in leading order (LO) only the photongluon fusion (PGF) process [6]

$$
\vec{\gamma} \vec{g} \rightarrow Q \bar{Q}
$$

contributes (an arrow denotes a longitudinally polarized particle). All studies of this process so far [5, 6, 7, 8] were limited to LO estimates which, however, are known to be notoriously unreliable due to, e.g., their strong factorization/renormalization scale dependence. In addition, the already available unpolarized NLO corrections [9, 10] turn out to be sizeable in certain kinematical regions and the 'clean picture' of Eq. (1) is obscured by genuine NLO subprocesses with light quarks in the initial state. The knowledge of the polarized NLO cross section is thus mandatory for a meaningful extraction of $\Delta g$. It is the main purpose of this paper to provide for the first time the results of such a complete NLO $\left(\mathcal{O}\left(\alpha_{s}^{2} \alpha\right)\right)$ QCD calculation. Thereby we hope to provide a more reliable theoretical basis for the measurement of $\Delta g$ by the COMPASS collaboration.

The remainder of the paper is organized as follows: next we briefly sketch the most 
important details of our calculation, mainly concentrating on complications which arise due to the presence of polarized particles in the initial state. We then apply our results to present for the first time estimates for the longitudinal spin asymmetry of total open charm photoproduction in NLO QCD in the kinematical region accessible by the COMPASS experiment [5]. Finally, we address the relevance of the main theoretical uncertainties inherent in this calculation. For more details on our calculation, analytical NLO results, and studies of $p_{T}$ and rapidity differential heavy quark cross sections, we refer the interested reader to Ref. [11].

The NLO QCD corrections to the PGF mechanism in Eq. (11) consist of three parts:

(i) the one-loop virtual corrections,

(ii) the real corrections with an additional gluon in the final state

$$
\vec{\gamma} \vec{g} \rightarrow Q \bar{Q} g
$$

(iii) a new production mechanism appearing for the first time in NLO

$$
\vec{\gamma} \vec{q}(\overrightarrow{\bar{q}}) \rightarrow Q \bar{Q} q(\bar{q})
$$

As is well known one encounters various types of singularities when calculating the loopand $2 \rightarrow 3$ phase space integrals. Ultraviolet (UV) singularities which show up only in the virtual corrections (i) are removed by on-shell mass and wavefunction and (modified) $\overline{\mathrm{MS}}$ coupling constant renormalization. In the latter case one also removes the gluon selfenergy contribution due to the heavy quark loop in addition to the subtractions usually performed in the $\overline{\mathrm{MS}}$ scheme (see, e.g., [9, 11, 12]). This explicit decoupling of the heavy quark at low energy leads to a fixed flavor scheme with $n_{l f}$ light flavors (active in the running of $\alpha_{s}$ and in the parton evolution) and the produced heavy flavor. Infrared (IR) divergencies and double pole terms appearing in the non-abelian parts when IR and mass/collinear (M) singularities coincide cancel in the sum of (i) and (ii). Finally one is left with the $\mathrm{M}$ divergencies in (ii) and (iii) which are removed by the factorization procedure in the $\overline{\mathrm{MS}}$ scheme. 
One has to choose a consistent method of regularizing these singularities so that they become manifest. For this purpose we choose to work in the well-established framework of $n$-dimensional regularization. When calculating the required helicity dependent matrix elements we have to project onto the helicity states of the incoming particles. This is achieved by using the standard relations (see, e.g., [13])

$$
\epsilon_{\mu}\left(k_{1}, \lambda_{1}\right) \epsilon_{\nu}^{*}\left(k_{1}, \lambda_{1}\right)=\frac{1}{2}\left[-g_{\mu \nu}+i \lambda_{1} \epsilon_{\mu \nu \rho \sigma} \frac{k_{1}^{\rho} k_{2}^{\sigma}}{k_{1} \cdot k_{2}}\right]
$$

for incoming photons with momentum $k_{1}$ and helicity $\lambda_{1}$ (accordingly for gluons with $k_{2}$ and $\left.\lambda_{2}\right)$ and

$$
u\left(k_{2}, h\right) \bar{u}\left(k_{2}, h\right)=\frac{1}{2} \not k_{2}\left(1-h \gamma_{5}\right)
$$

for incoming quarks with momentum $k_{2}$ and helicity $h$ (analogously for antiquarks). Using (4) and (5) we can calculate the contributions to heavy flavor photoproduction with unpolarized and polarized beams simultaneously by taking the sum or the difference of the helicity dependent squared matrix elements

$$
\begin{aligned}
& \text { unpolarized : } \overline{|\mathrm{M}|}^{2}=\frac{1}{2}\left[|M|^{2}(++)+|M|^{2}(+-)\right] \\
& \text { polarized : } \Delta|\mathrm{M}|^{2}=\frac{1}{2}\left[|M|^{2}(++)-|M|^{2}(+-)\right]
\end{aligned}
$$

where $|M|^{2}\left(h_{1}, h_{2}\right)$ denotes the squared matrix element for any of the contributing subprocesses (11) and (i)-(iii) for definite helicities $h_{1}$ and $h_{2}$ of the two incoming particles. The possibility to obtain the unpolarized results" 'for free' provides an important check on the correctness of our results. We fully agree with the unpolarized results presented in [9] and [10]. The abelian part of our result agrees analytically with the results of two recent calculations of the NLO QCD corrections to $\vec{\gamma} \vec{\gamma} \rightarrow Q \bar{Q}$ [14] as well.

The presence of $\gamma_{5}$ and the totally anti-symmetric tensor $\epsilon_{\mu \nu \rho \sigma}$ in the polarized calculation due to (4) and (5) introduces some complications because their purely fourdimensional origin allows no straightforward continuation to $n \neq 4$ dimensions. We choose to work in the HVBM scheme [15] which was shown to provide an internally consistent

\footnotetext{
${ }^{1}$ Since each boson has $n-2$ degrees of freedom, one has to average the spin of each incoming photon (gluon) with a factor $1 /(n-2)$ replacing the $1 / 2$ in (4) for the unpolarized case. Furthermore, the inclusion of external ghost contributions (see, e.g., [11]) allows us to drop all terms other than $g_{\mu \nu}$ in the symmetric part of (4).
} 
extension of $\gamma_{5}$ and $\epsilon_{\mu \nu \rho \sigma}$ to arbitrary dimensions ${ }^{2}$. The price to pay is that apart from the usual $n$-dimensional scalar products $k \cdot p$ their respective $(n-4)$-dimensional subspace counterparts, usually denoted by $\widehat{k \cdot p}$ ('hat momenta'), can also show up in $|M|^{2}\left(h_{1}, h_{2}\right)$ in the HVBM scheme. For the single-inclusive heavy quark production considered here, one can choose a convenient frame (see [11] for details) where all non-vanishing $(n-4)$ scalar products can be expressed by a single hat momenta combination $\hat{p}^{2}=-\widehat{p \cdot p}$. The latter quantity appears exclusively in the polarized $2 \rightarrow 3$ contributions and originates from scalar products of the momenta of the two not observed (integrated) final state particles in (2). However, terms proportional to $\hat{p}^{2}$ still deserve special attention when performing the $2 \rightarrow 3$ phase space integrations since the $(n-4)$-dimensional subspace cannot be trivially integrated out as in an unpolarized calculation.

The appropriately modified phase space formula for the present polarized calculation can be schematically expressed as follows

$$
\mathrm{dPS}_{3}=\mathrm{dPS}_{3, \text { unp }}\left(\theta_{1}, \theta_{2}\right) \times \frac{1}{\mathrm{~B}\left(\frac{1}{2}, \frac{\mathrm{n}-4}{2}\right)} \int_{0}^{1} \mathrm{dx} \frac{\mathrm{x}^{(\mathrm{n}-6) / 2}}{\sqrt{1-\mathrm{x}}}
$$

with

$$
x \equiv \hat{p}^{2} / \hat{p}_{\max }^{2}=\frac{4\left(s_{4}+m^{2}\right) \hat{p}^{2}}{s_{4}^{2} \sin ^{2} \theta_{1} \sin ^{2} \theta_{2}}
$$

where $B$ is the Euler Beta function, $m$ denotes the mass of the heavy quark, and $s_{4} \equiv$ $s+t_{1}+u_{1}$ is the sum of the three $2 \rightarrow 2$ Mandelstam variables. The angles $\theta_{1,2}$ are introduced to parametrize the momenta of the two integrated partons (see [11]). Since

$$
\frac{1}{B\left(\frac{1}{2}, \frac{n-4}{2}\right)} \int_{0}^{1} d x \frac{x^{(n-6) / 2}}{\sqrt{1-x}}\left\{\begin{array}{c}
1 \\
\hat{p}^{2}
\end{array}=\left\{\begin{array}{c}
1 \\
(n-4) s_{4}^{2} \sin ^{2} \theta_{1} \sin ^{2} \theta_{2} /\left[4\left(s_{4}+m^{2}\right)\right]
\end{array},\right.\right.
$$

Eq. (8) reduces to the well-known 'unpolarized' phase space $\mathrm{dPS}_{3, \text { unp }}$ (see, e.g., [17]) for the vast majority of terms without a $\hat{p}^{2}$ dependence and for the rest, which is proportional to $\hat{p}^{2}$, introduces a $n \rightarrow n+2$ shift in the angular integrals (11) discussed below, guaranteeing collinear safety. Due to the extra powers of $s_{4}$ all these additional integrals are infrared safe as well, implying that all contributions due to $\hat{p}^{2}$ are at least of $\mathcal{O}(n-4)$ (cf. Eq. (10)) and hence drop out when the limit $n \rightarrow 4$ is taken?.

\footnotetext{
${ }^{2}$ This prescription is incorporated in TRACER 16], which we have used for all trace calculations.

${ }^{3}$ This differs from a calculation [18] involving only massless particles, where IR poles can occur.
} 
The remaining phase space integration then proceeds as in the unpolarized case [9]. One has to make extensive use of relations between Mandelstam variables to reduce complex combinations to simpler ones by partial fractioning. In the end only integrals containing at most two angular dependent Mandelstam variables remain

$$
I^{(k, l)}=\int_{0}^{\pi} d \theta_{1} \int_{0}^{\pi} d \theta_{2} \frac{\sin ^{n-3} \theta_{1} \sin ^{n-4} \theta_{2}}{\left(a+b \cos \theta_{1}\right)^{k}\left(A+B \cos \theta_{1}+C \sin \theta_{1} \cos \theta_{2}\right)^{l}}
$$

where $a, b, A, B$, and $C$ are only functions of $s, t_{1}, u_{1}$, and $m^{2}$. A useful list of integrals of the type (11) can be found in [17], but we have checked all required formulae. The loop integrations have been performed using a Passarino-Veltman reduction [19] to scalar integrals which can also be found in [17] and we have recalculated them as well.

In the soft gluon limit for (2) the $2 \rightarrow 3$ kinematics reduces to the usual $2 \rightarrow 2$ Born kinematics and soft gluon poles associated with $1 / s_{4}$ terms occur, since $s_{4} \rightarrow 0$ when the final state gluon momentum $k_{3}$ becomes soft $\left(k_{3} \rightarrow 0\right)$. To deal with these poles one can simply slice the phase space into a soft gluon $\left(s_{4}<\Delta\right)$ and a hard gluon $\left(s_{4}>\Delta\right)$ part [9], where $\Delta$ has to be much smaller than $s, t_{1}, u_{1}$ and $m^{2}$. The soft gluon cross section, related to $\int_{0}^{\Delta} d s_{4}$, can then be obtained analytically [11] and the singularities cancel in the sum with the virtual cross sections whereas the hard gluon part contains only the left over M singularities which are removed by factorization.

As our final technical remark let us recall that the factorization 'counter cross section' for the light quark initiated subprocess (3) contains a part which can be schematically expressed as

$$
d \Delta \sigma_{q \gamma}^{\mathrm{fact}}=-\frac{\alpha_{s}}{2 \pi}\left[\left(\frac{2}{n-4} \Delta P_{q \gamma}+\Delta F_{q \gamma}\right) \otimes d \Delta \sigma_{q \bar{q} \rightarrow Q \bar{Q}}\right]+\ldots
$$

where $\otimes$ denotes a convolution, $\Delta P_{q \gamma}$ is the usual LO spin-dependent photon-to-quark splitting function and $\Delta F_{i \gamma}$ represents the freedom in choosing a factorization prescription. In the $\overline{\mathrm{MS}}$ scheme (which we use) $\Delta F_{q \gamma}$ has the form

$$
\Delta F_{q \gamma}^{\overline{\mathrm{MS}}}\left(x, \mu_{f}, \mu\right)=\Delta P_{q \gamma}(x)\left(\gamma_{E}-\ln 4 \pi+\ln \frac{\mu_{f}^{2}}{\mu^{2}}\right)
$$

where $\gamma_{E}$ is the Euler constant, $\mu$ is the mass scale introduced by $n$ dimensional regularization, and $\mu_{f}$ is the arbitrary factorization scale (in other schemes one subtracts 
different terms in (13)). By applying (12) one introduces the parton content of the real (on-shell) polarized photon [20] which is experimentally completely unknown for the time being. Strictly speaking a physically consistent (i.e., scheme independent) result for heavy quark photoproduction in $\mathcal{O}\left(\alpha_{s}^{2} \alpha\right)$ can only be obtained for the sum of the 'direct' and 'resolved' contribution due to the freedom in (13). In the first case the photon acts as an elementary particle (as in the present calculation) whereas in the latter case it resolves into its parton content before the hard scattering takes place. The NLO corrections for the 'resolved' contribution are unknown so far for the polarized case and thus, for the time being, have to be estimated in LO (see below).

Let us now turn to some numerical results and phenomenological aspects. We are here only interested in the experimentally most relevant total photoproduction cross section (for other results see [11]). Let us start with the total photon-parton cross section in NLO which can be expressed in terms of scaling functions $(i=g, q, \bar{q})$

$$
\Delta \hat{\sigma}_{i \gamma}\left(s, m^{2}, \mu_{f}\right)=\frac{\alpha \alpha_{s}}{m^{2}}\left[\Delta f_{i \gamma}^{(0)}(\eta)+4 \pi \alpha_{s}\left\{\Delta f_{i \gamma}^{(1)}(\eta)+\Delta \bar{f}_{i \gamma}^{(1)}(\eta) \ln \frac{\mu_{f}^{2}}{m^{2}}\right\}\right]
$$

where $\Delta f_{i \gamma}^{(0)}$ and $\Delta f_{i \gamma}^{(1)}, \Delta \bar{f}_{i \gamma}^{(1)}$ stand for the LO and NLO corrections, respectively, $\mu_{f}$ denotes the factorization scale, and $\eta \equiv s / 4 m^{2}-1$. Note that for simplicity we have made the conventional choice [9] $\mu_{r}=\mu_{f}$ in (14) and in what follows (see [11] for an independent variation of $\mu_{f}$ and $\mu_{r}$ ). The scaling functions can be further decomposed depending on the electric charge of the heavy and light quarks, $e_{Q}$ and $e_{q}$, respectively:

$$
\begin{aligned}
\Delta f_{g \gamma}(\eta) & =e_{Q}^{2} \Delta c_{g \gamma}(\eta) \\
\Delta f_{q \gamma}(\eta) & =e_{Q}^{2} \Delta c_{q \gamma}(\eta)+e_{q}^{2} \Delta d_{q \gamma}(\eta)
\end{aligned}
$$

with corresponding expressions for the $\Delta \bar{f}_{i \gamma}$.

In Fig. 1 we present $\Delta c_{g \gamma}^{(0)}, \Delta c_{g \gamma}^{(1)}$, and $\Delta \bar{c}_{g \gamma}^{(1)}$ as a function of the scaling parameter $\eta$ in the $\overline{\mathrm{MS}}$ scheme (the threshold $s=4 m^{2}$ is located at $\eta=0$ ). Also shown (dotted line) is the contribution to $\Delta c_{g \gamma}^{(1)}$ from the hard gluon part $\left(s_{4}>\Delta\right)$ alone. From a comparison

\footnotetext{
${ }^{4}$ Note that the interference term of the two possible production mechanisms for the subprocess (3) proportional to $e_{Q} e_{q}$ does not contribute to $\Delta f_{q \gamma}$ in (16), since it vanishes when integrated over the entire phase space as a consequence of the Furry theorem.

${ }^{5}$ To define this quantity we follow the procedure in [9] and explicitly add the $\Delta$ dependent terms
} 
of this dotted curve with the dashed one showing the full NLO coefficient $\Delta c_{g \gamma}^{(1)}$ (i.e., the sum of the hard and soft plus virtual contributions), one can infer that the soft plus virtual contribution is almost negligible for $\eta \lesssim 1$. For the interpretation of the results below it is important to notice that the LO coefficient (solid line) in Fig. 1 changes sign at $\eta \simeq 3$. Upon adding the NLO contributions, multiplied by a factor $4 \pi \alpha_{s}$ (see Eq. (14)), the zero is shifted towards $\eta \simeq 1$. We also note that for $\eta \lesssim 0.1$ the $\mathcal{O}\left(\alpha_{s}\right)$ corrections dominate over the Born approximation when we include that factor. This enhancement near threshold is due to large logarithms from initial state gluon bremsstrahlung [9]. Furthermore in the threshold limit $\eta \rightarrow 0$ the soft plus virtual piece tends to a constant ('Coulomb singularity') while in LO $\Delta c_{g \gamma}^{(0)} \rightarrow 0$.

Fig. 2 compares in a similar way the light quark induced coefficients $\Delta c_{q \gamma}^{(1)}, \Delta \bar{c}_{q \gamma}^{(1)}, \Delta d_{q \gamma}^{(1)}$, and $\Delta \bar{d}_{q \gamma}^{(1)}$ as a function of $\eta$ in the $\overline{\mathrm{MS}}$ scheme. Numerically they turn out to be much smaller than their gluonic counterparts shown in Fig. 1. Comparing our polarized and unpolarized results (for the latter see also Figs. 5, 7, and 8 in [9]) for either the gluon or the quark coefficients, we find the same pattern: for $\eta \rightarrow 0$ the unpolarized and polarized results become equal, e.g., $\Delta c_{g \gamma} \rightarrow c_{g \gamma}$. A glance at Eqs. (6) and (77) immediately implies that $\left|M_{i \gamma}\right|^{2}(+-) \rightarrow 0$. On the contrary, for asymptotically large energies $\eta \rightarrow \infty$ the unpolarized NLO coefficients approach a large plateau value, except for $d_{q \gamma}^{(1)}$ and $\bar{d}_{q \gamma}^{(1)}$, dominating over the LO result due to Feynman diagrams with a gluon exchange in the t-channel [9] while all polarized coefficients tend to zero in that kinematical region. Thus one can infer that here $\left|M_{i \gamma}\right|^{2}(++) \rightarrow\left|M_{i \gamma}\right|^{2}(+-)$.

With the total partonic cross section in (14) at hand is is now straightforward to calculate the total hadronic heavy flavor photoproduction cross section via

$$
\Delta \sigma_{\gamma p}^{Q}\left(S_{\gamma p}, m^{2}, \mu_{f}\right)=\sum_{f=q, \bar{q}, g_{4 m^{2} / S_{\gamma p}}} \int_{1}^{1} d x \Delta \hat{\sigma}_{f \gamma}\left(x S_{\gamma p}, m^{2}, \mu_{f}\right) \Delta f^{p}\left(x, \mu_{f}^{2}\right)
$$

depending on the available photon-proton c.m.s. energy $S_{\gamma p}$. Of course an expression similar to (17) also holds for the unpolarized cross section $\sigma_{\gamma p}^{Q}$ with all polarized quantities replaced by the corresponding unpolarized ones. Instead of measuring $\Delta \sigma_{\gamma p}^{Q}$ in (17) $\left(\sim \ln \Delta / m^{2}\right)$ of the soft plus virtual cross section to the hard part in order to cancel the dependence on the auxiliary quantity $\Delta$ numerically. 
directly (which requires the determination of the absolute normalization), experiments will study the related longitudinal spin asymmetry defined by

$$
A_{\gamma p}^{Q}\left(S_{\gamma p}, m^{2}, \mu_{f}\right)=\frac{\Delta \sigma_{\gamma p}^{Q}\left(S_{\gamma p}, m^{2}, \mu_{f}\right)}{\sigma_{\gamma p}^{Q}\left(S_{\gamma p}, m^{2}, \mu_{f}\right)} .
$$

In Fig. 3 we show the LO and NLO predictions for the total charm asymmetry $A_{\gamma p}^{c}$, using $m=1.5 \mathrm{GeV}, \mu_{f}=2 m$, and three different sets of polarized parton distributions [2, 3] which mainly differ in $\Delta g$, in the $\sqrt{S_{\gamma p}}$ region accessible by the upcoming COMPASS experiment at CERN [5]. Depending on the used muon beam energy (100 or $200 \mathrm{GeV}$ ) they will determine $A_{\gamma p}^{c}$ for one average value of $\sqrt{S_{\gamma p}}$ of about $10 \mathrm{GeV}$. The NLO corrections in Fig. 3 turn out to be sizeable, depend strongly on $\sqrt{S_{\gamma p}}$ and do not cancel in the ratio (18) as one may naively expect. However, the physical origin of these large corrections is readily explained in terms of the LO and NLO coefficient functions (see Fig. 1 and 2).

For $\sqrt{S_{\gamma p}} \simeq 10 \mathrm{GeV}$ one probes $\eta$ values from threshold up to $\eta \approx 10$. Note that in $\Delta \sigma_{\gamma p}^{c}$, see Eq. (17), the coefficient functions for small values of $\eta$ (i.e., small $s=x S_{\gamma p}$ ) are convoluted with the gluon distribution at the smallest possible $x\left(x \geq 4 m^{2} / S_{\gamma p}\right)$ where $\Delta g$ is usually large and vice versa. Since the NLO corrections to the gluonic coefficient functions, which dominate for small $\eta$, decrease faster with increasing $\eta$ in the polarized case due to the zero, one should expect the NLO asymmetry to be somewhat smaller than the LO one in that particular $\sqrt{S_{\gamma p}}$ region if the LO and NLO $\Delta g$ are not too different. Moreover, the NLO shift of the zero towards smaller $\eta$ adds contributions with opposite sign in the convolution already for smaller $S_{\gamma p}$. This should also lower the NLO asymmetry with respect to the LO one for not too large $S_{\gamma p}$. Instead we find (see Fig. 3) that the NLO asymmetries for GRSV [2] and GS (A) [3] are larger than the LO ones for small $S_{\gamma p}$. But this is entirely due to the badly constrained $\Delta g$ at large $x$ and thus should not be taken too seriously: For $\sqrt{S_{\gamma p}} \simeq 10 \mathrm{GeV}$ the convolution (17) samples $x \gtrsim 0.1$ and in both sets of polarized parton distributions [2, 3] the NLO $\Delta g$ is much larger than the LO one (especially in the case of GS (A)). This is not the case for the unpolarized GRV distributions [21] which we use to calculate $\sigma_{\gamma p}^{c}$ in (18). If we instead use NLO gluons for both the NLO and LO asymmetries, we find the expected behaviour discussed above 
for GRSV and GS (A). This is demonstrated for the GRSV set by the dotted curve in Fig. 3. The situation for GS (C) [3], however, is even more complex since in this case the gluon oscillates as well in the covered $x$ region. Also obviously the relative NLO corrections (' $K$-factors') are always very large around zeros in the LO asymmetry, as for the GS (C) curve in Fig. 3 at around $\sqrt{S_{\gamma p}} \simeq 12 \mathrm{GeV}$ (similarly for GS (A) and GRSV at larger $\sqrt{S_{\gamma p}} \simeq 30 \mathrm{GeV}$ not shown in the figure), but this is natural for quantities that can change sign.

Let us finally briefly discuss the importance of the main theoretical uncertainties in the calculation of $\Delta \sigma_{\gamma p}^{c}$ or $A_{\gamma p}^{c}$ which may further complicate the extraction of $\Delta g$. First of all Fig. 4 shows the dependence of $\Delta \sigma_{\gamma p}^{c}$ on the choice of the factorization scale in the relevant region $\mu_{f}^{2}=\left[m^{2}, \ldots, 4 m^{2}\right]$ for the GRSV standard set of polarized parton densities [2] for two different values of $\sqrt{S_{\gamma p}}$. The NLO results hardly depend on the precise choice of $\mu_{f}$ whereas the LO cross sections vary by as much as $50 \%$ in the shown range. This clearly underlines the importance (and usefulness) of the full NLO calculation. NLO results for $\Delta \sigma_{\gamma p}^{c}$ or $A_{\gamma p}^{c}$ are thus much more trustworthy than previous LO estimates.

As already mentioned above another complication in the extraction of $\Delta g$ arises due to the presence of the light quark induced subprocesses (3) which may serve as an important 'background'. We have checked that for $\sqrt{S_{\gamma p}} \simeq 10 \mathrm{GeV}$ this contribution is fairly small (about 5\%, except for the GS (C) set where the gluon contribution is close to zero at this $\sqrt{S_{\gamma p}}$ ) and can possibly be neglected for a first determination of $\Delta g$. However, with a better knowledge of the polarized quark densities in the future the light quark contribution should be subtracted. A similar remark holds for the importance of the possible 'background' from 'resolved' photons. Since the parton content of polarized photons is completely unknown so far, one has to impose some realistic models [20] to estimate this contribution. In [7] it was shown that even for rather large photonic densities this 'background' should also be very small in the 'COMPASS region'. Moreover, the unknown precise value of the charm quark mass $m$ leads to shifts in $\Delta \sigma_{\gamma p}$ and $A_{\gamma p}^{c}$ of about $30 \%$ when $m$ is varied by $0.2 \mathrm{GeV}$ around the central values of $1.5 \mathrm{GeV}$ used in Figs. 3 and 4. 
Finally, it should be stressed that for a meaningful and reliable extraction of $\Delta g$ from a measurement of $A_{\gamma p}^{c}$ (i.e., roughly a measurement of $\Delta g / g$ ) our knowledge of the

unpolarized gluon density has to be improved as well. At $\sqrt{S_{\gamma p}} \simeq 10 \mathrm{GeV}$ one probes the gluon at $x$ values larger than 0.1 where the uncertainty in $g\left(x, \mu^{2}\right)$ is sizeable and nonnegligible (see, e.g., [22, 23]). In that particular $x$ region $g\left(x, \mu^{2}\right)$ is mainly constrained by direct photon data, but with rather large theoretical uncertainties [24]. Ideally, COMPASS should try to measure also the unpolarized charm photoproduction cross section, thereby reducing also our present ignorance on $g$ at large $x$. Anyway, improvements seem to be mandatory for a reliable extraction of $\Delta g$.

To summarize, we have presented the main results of a first complete NLO QCD calculation for heavy flavor photoproduction with longitudinally polarized beams. The NLO corrections are sizeable for the total longitudinal spin asymmetry in the energy range accessible by the forthcoming COMPASS experiment. But these corrections can be partly attributed to the rather different large $x$ behaviour in LO and NLO of the presently available sets of polarized parton densities, which only reflects our complete ignorance of $\Delta g$. The theoretical uncertainties associated with the precise choice of a factorization scale were shown to be strongly reduced in NLO. The prospects for a first direct measurement of the polarized gluon density still seem to be promising but a careful and reliable extraction of $\Delta g$ requires also an improved knowledge of the unpolarized gluon density at large $x$ and of the precise value of the charm quark mass. Uncertainties due to the 'background' from 'resolved' photons or light quark induced processes should to be of less importance.

\section{Acknowledgments}

We are grateful to W. Vogelsang for useful discussions. I.B. wishes to thank E. Reya for suggesting this problem and his constant encouragement, and W. Beenakker for helpful comments on some integrals. This work has been supported in part by the 'Bundesministerium für Bildung, Wissenschaft, Forschung und Technologie', Bonn. 


\section{References}

[1] A recent overview of the experimental status can be found for example in the proceedings of the workshop 'Deep Inelastic Scattering off Polarized Targets: Theory Meets Experiment', Zeuthen, Germany, 1997, ed. by J. Blümlein et al., DESY 97-200, pp. $2-40$.

[2] M. Glück, E. Reya, M. Stratmann, and W. Vogelsang, Phys. Rev. D53, 4775 (1996).

[3] T. Gehrmann and W.J. Stirling, Phys. Rev. D53, 6100 (1996).

[4] G. Altarelli, R.D. Ball, S. Forte, and G. Ridolfi, Nucl. Phys. B496, 337 (1997), CERN-TH-98-61, hep-ph/9803237;

D. de Florian, O. Sampayo, and R. Sassot, Phys. Rev. D57, 5803 (1998);

E. Leader, A.V. Sidorov, and D.B. Stamenov, hep-ph/9708335;

K. Abe et al., E154 collab., Phys. Lett. B405, 180 (1997);

D. Adams et al., SMC, Phys. Rev. D56, 5330 (1997).

[5] G. Baum et al., COMPASS collab., CERN/SPSLC-96-14, CERN/SPSLC-96-30.

[6] M. Glück and E. Reya, Z. Phys. C39, 569 (1988).

[7] M. Stratmann and W. Vogelsang, Z. Phys. C74, 641 (1997).

[8] G. Altarelli and W.J. Stirling, Particle World 1, 40 (1989);

M. Glück, E. Reya, and W. Vogelsang, Nucl. Phys. B351, 579 (1991);

S.I. Alekhin, V.I. Borodulin, and S.F. Sultanov, Int. J. Mod. Phys. A8, 1603 (1993);

S. Keller and J.F. Owens, Phys. Rev. D49, 1199 (1994);

S. Frixione and G. Ridolfi, Phys. Lett. B383, 227 (1996).

[9] J. Smith and W.L. van Neerven, Nucl. Phys. B374, 36 (1992).

[10] R.K. Ellis and P. Nason, Nucl. Phys. B312, 551 (1989).

[11] I. Bojak and M. Stratmann, in preparation.

[12] P. Nason, S. Dawson, and R.K. Ellis, Nucl. Phys. B303, 607 (1988), B327, 49 (1989). 
[13] N.S. Craigie, K. Hidaka, M. Jacob, and F.M. Renard, Phys. Rep. 99, 69 (1983).

[14] B. Kamal, Z. Merebashvili, and A.P. Contogouris, Phys. Rev. D51, 4808 (1995), D55, 3229(E) (1997);

G. Jikia and A. Tkabladze, Phys. Rev. D54, 2030 (1996).

[15] G. t'Hooft and M. Veltman, Nucl. Phys. B44, 189 (1972);

P. Breitenlohner and D. Maison, Comm. Math. Phys. 52, 11 (1977).

[16] M. Jamin and M.E. Lautenbacher, Comput. Phys. Commun. 74, 265 (1993).

[17] W. Beenakker, H. Kuijf, W.L. van Neerven, and J. Smith, Phys. Rev. D40, 54 (1989).

[18] L.E. Gordon and W. Vogelsang, Phys. Rev. D48, 3136 (1993).

[19] G. Passarino and W. Veltman, Nucl. Phys. B160, 151 (1979);

W.J.P. Beenakker, Ph.D. thesis, Univ. Leiden.

[20] M. Glück and W. Vogelsang, Z. Phys. C55, 353 (1992), C57, 309 (1993);

M. Glück, M. Stratmann, and W. Vogelsang, Phys. Lett. B337, 373 (1994);

M. Stratmann and W. Vogelsang, Phys. Lett. B386, 370 (1996).

[21] M. Glück, E. Reya, and A. Vogt, Z. Phys. C67, 433 (1995).

[22] J. Huston et al., CTEQ collab., FERMILAB-PUB-98-046-T, hep-ph/9801444.

[23] A.D. Martin, R.G. Roberts, W.J. Stirling, and R.S. Thorne, hep-ph/9803445.

[24] W. Vogelsang and A. Vogt, Nucl. Phys. B453, 334 (1995). 


\section{Figure Captions}

Fig. 1 The LO and NLO gluonic scaling functions in the $\overline{\mathrm{MS}}$ scheme as a function of $\eta=s / 4 m^{2}-1$ as defined in Eqs. (14) - (16).

Fig. 2 Same as in Fig. 1, but for the NLO light quark coefficient functions.

Fig. 3 The longitudinal spin asymmetry for total charm quark photoproduction in LO and NLO as defined in (18) for $m=1.5 \mathrm{GeV}, \mu_{f}=2 m$ and three different sets of LO and NLO polarized parton densities [2, 3]. The unpolarized cross section in (18) was calculated using the GRV [21] densities. Also shown (dotted line) is the LO asymmetry using the NLO GRSV [2] gluon distribution (see text). The vertical bar shows the estimated statistical uncertainty $\delta A_{\gamma p}^{c}$ for such a measurement at COMPASS [5].

Fig. 4 The factorization scale dependence for the LO and NLO polarized total charm quark photoproduction cross section $\Delta \sigma_{\gamma p}^{c}$ as defined in (17) using the LO and NLO GRSV standard densities [2], respectively, for $m=1.5 \mathrm{GeV}$. 


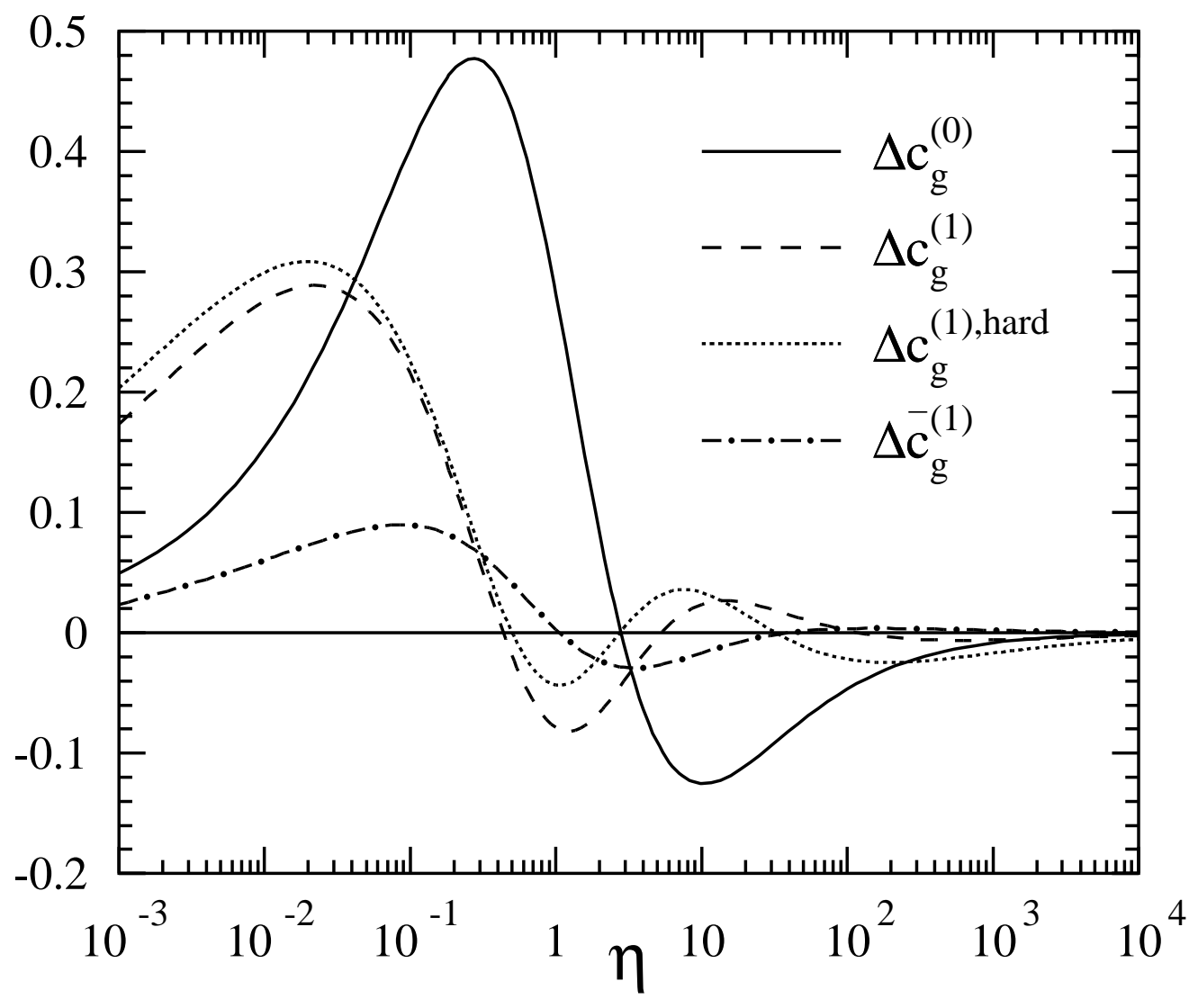

Fig. 1 


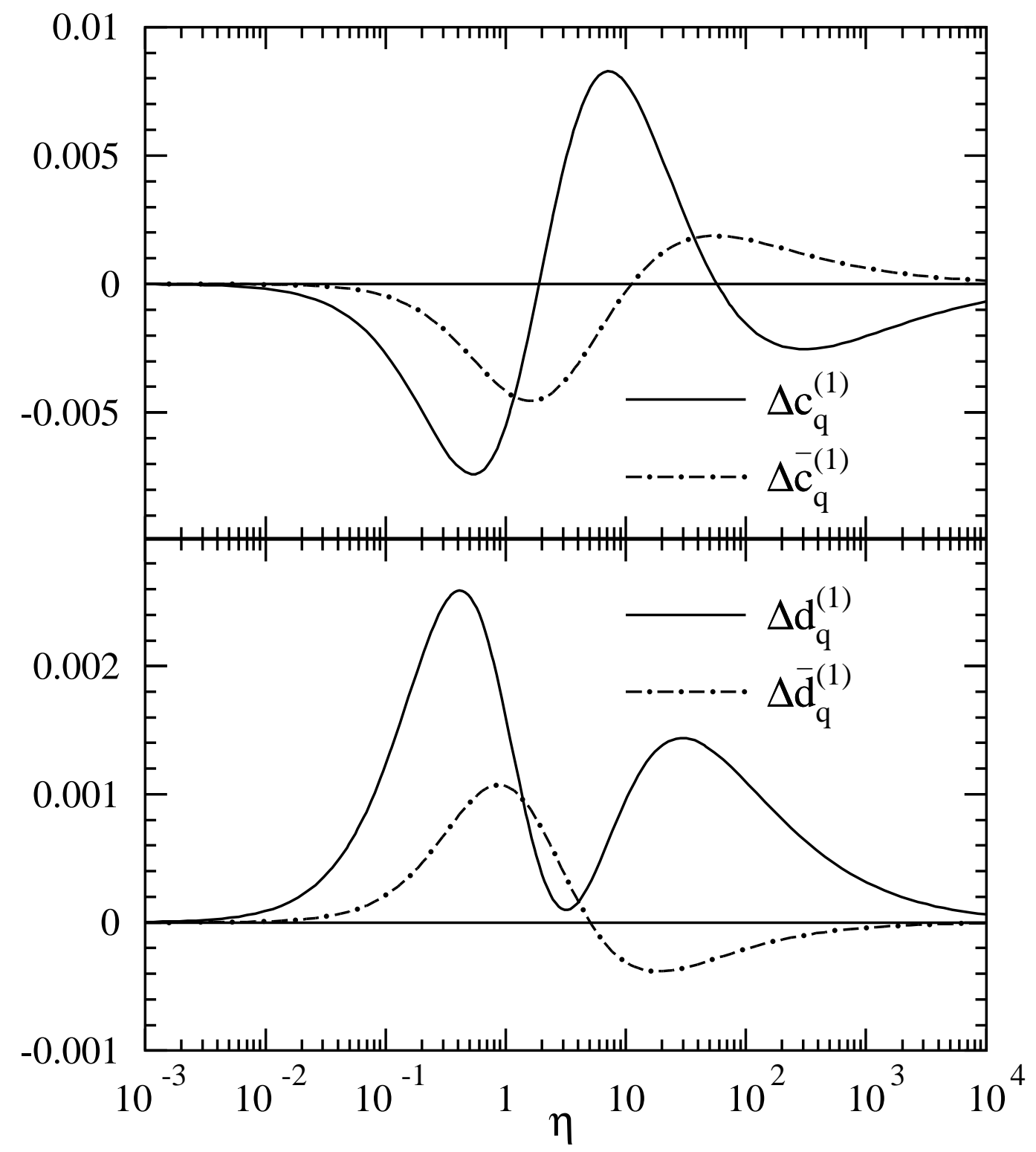

Fig. 2 


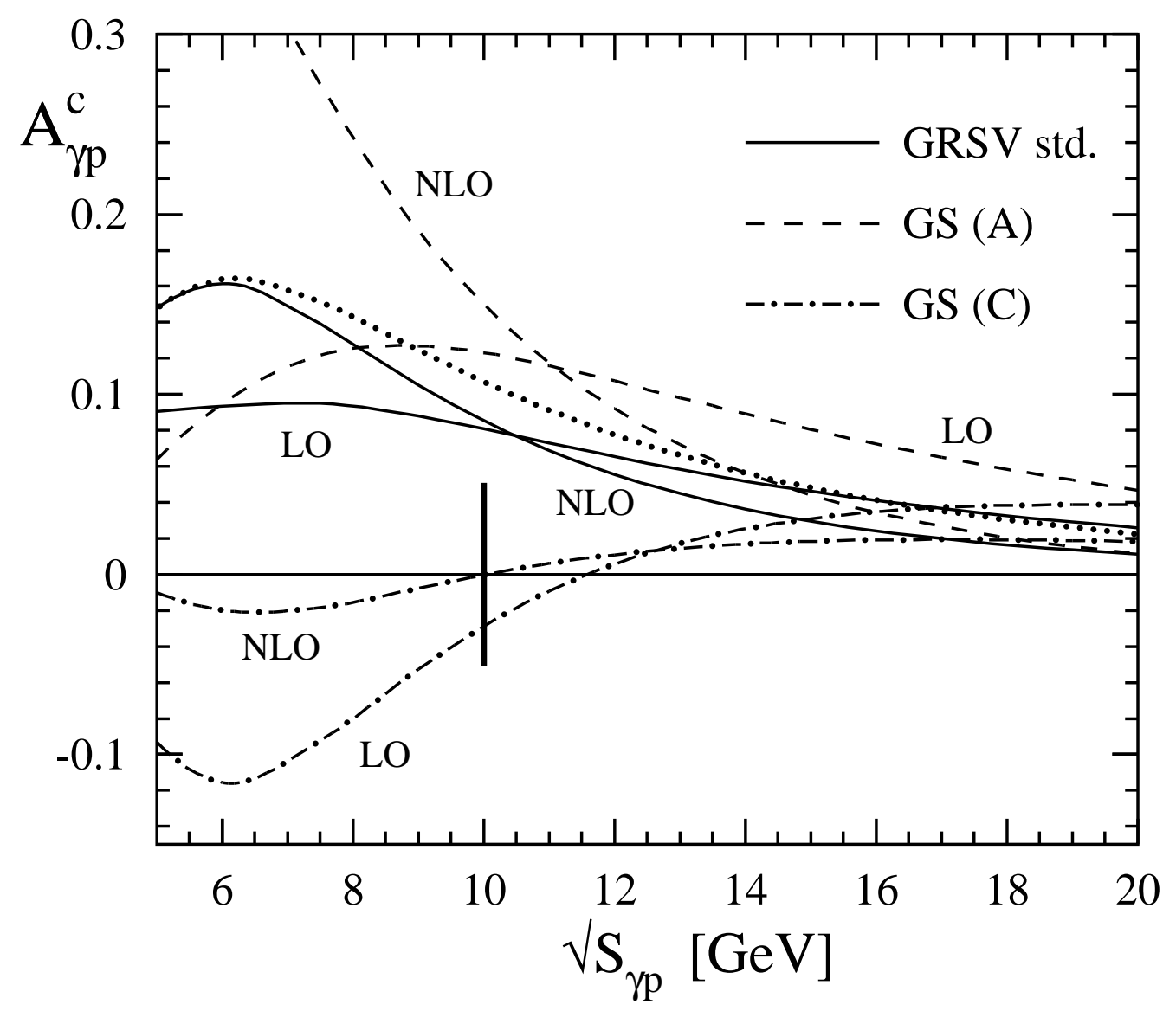

Fig. 3 


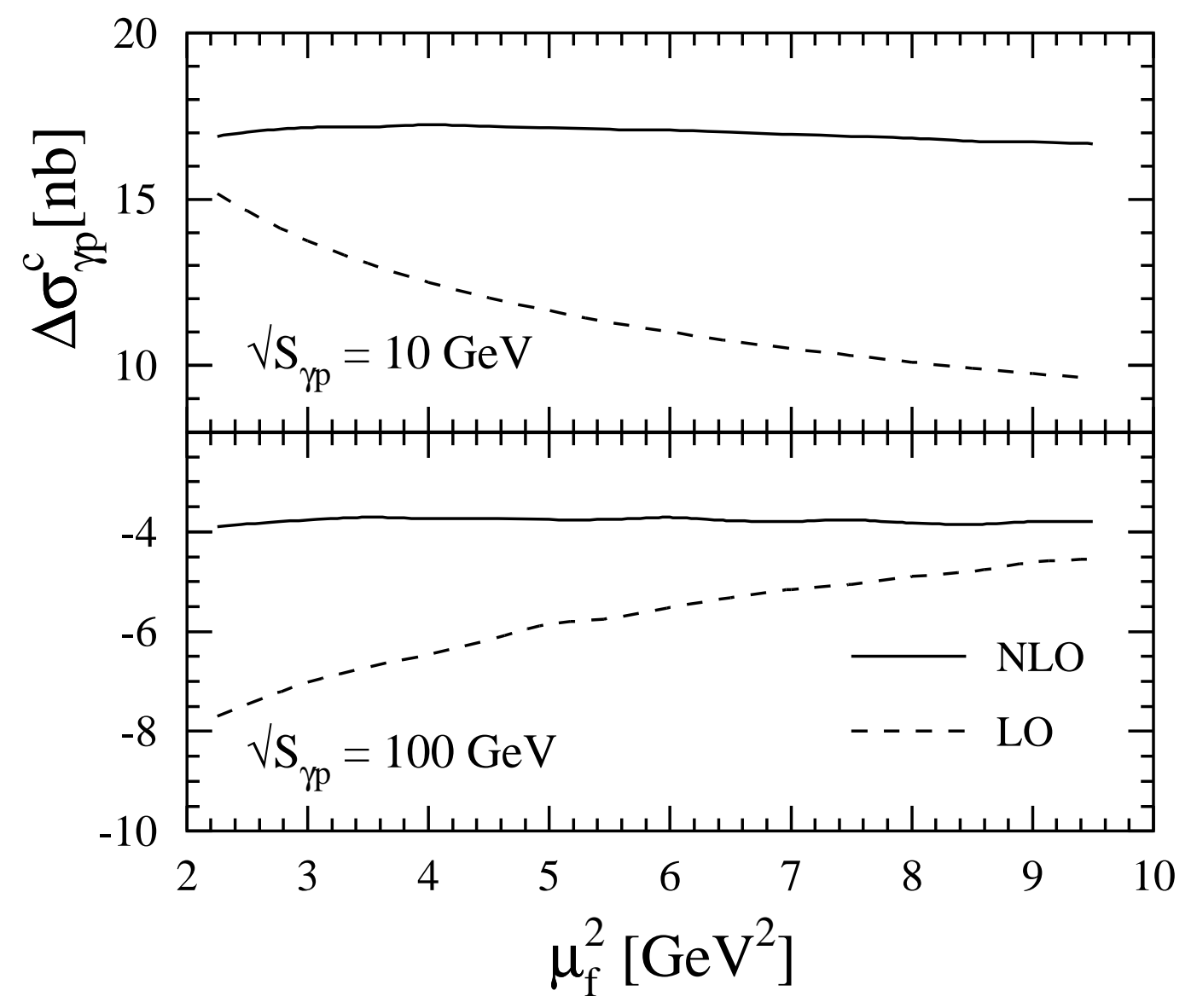

Fig. 4 